\title{
Shear waves induced by moving needle in MR Elastography
}

\author{
Q. C. C. Chan ${ }^{1}$, G. Li ${ }^{2}$, R. L. Ehman ${ }^{3}$, P. J. Rossman ${ }^{3}$, G. Cao ${ }^{4}$, R. Li ${ }^{5}$, E. S. Yang ${ }^{1,2}$ \\ ${ }^{1}$ Department of Electrical and Electronic Engineering, The University of Hong Kong \\ ${ }^{2}$ The Jockey Club MRI Engineering Centre, The University of Hong Kong \\ ${ }^{3}$ Department of Diagnostic Radiology, Mayo Clinic and Foundation \\ ${ }^{4}$ GE Medical Systems Asia \\ ${ }^{5}$ Department of Diagnostic Radiology, St Paul's Hospital
}

\begin{abstract}
Magnetic Resonance Elastography (MRE) is a phase contrast-based method for observing shear wave propagation in a material to determine its stiffness. The objective of this study was to determine whether shear waves suitable for MRE can be induced using a moving acupuncture needle. Tissue-simulating bovine gel phantom and a $0.4 \mathrm{~mm}$ diameter acupuncture needle were used in the experiment. The results showed that observable shear waves could be induced in the gel phantom by cyclic needle motion. The observed wavelength varied with excitation frequency, as expected. Generating shear waves using moving needles may be a useful tool to study the basic mechanism of acupuncture with MRE. Further study will be conducted to observe the wave motion in inhomogeneous media and acupuncture-induced effects in invivo studies.
\end{abstract} stiffness

Keywords-Acupuncture, MR Elastography, shear wave,

\section{INTRODUCTION}

Magnetic Resonance Elastography (MRE) is a phase contrast method used for quantitatively assessing the mechanical properties of tissue $[1,2]$. A mechanical actuator coupled to the tissue provides cyclic motion synchronized to the imaging sequence. Most previous studies have applied mechanical motion at the surface of the tissue [1-5]. Recently, Rossman et al. showed that longitudinal motion of a carbon fiber rod inserted into a $2 \%$ agarose gel phantom can generate shear waves [6]. The longitudinal motion (motion in the direction of the rod's length) was produced by a piezoelectric bending element. We hypothesize that it may be possible to study the basic mechanism of acupuncture in vivo using MRE by inserting acupuncture needles into muscle tissue. The purpose of this study was to determine whether or not shear waves could be produced in a phantom by longitudinal motion of an acupuncture needle.

\section{Methodology}

Fig. 1 depicts how the piezoelectric bending element is configured to produce longitudinal motion of the needle. The needle was $0.4 \mathrm{~mm}$ diameter and $50 \mathrm{~mm}$ long. Tissuesimulating bovine gel phantom (15\% bovine powder, approximately 225 bloom) was used in the experiment for observing the shear waves generated. MRE uses a modified phase-contrast MRI technique to image induced shear waves as they propagate through the phantom. In addition to standard MRI imaging gradients, the MRE sequence incorporates oscillating, motion sensitizing field gradients that are synchronized to the frequency of the propagating waves. Data acquisition parameters were: TR 100ms, TE min full, flip angle of $30^{\circ}, 256 \times 64$ acquisition matrix using a $16 \mathrm{~cm}$ axial field of view. The acquisition time for each scan was $76.8 \mathrm{~s}$. The excitation frequencies were $50 \mathrm{~Hz}, 100 \mathrm{~Hz}$ and $200 \mathrm{~Hz}$. The shear stiffness was calculated using the following equation:

$$
\mu=\rho f^{2} \lambda^{2}
$$

where $\rho$ is the density of the material, $f$ is the excitation frequency and $\lambda$ is the shear wave wavelength determined from the wave images. The density $\rho$ is assumed to be $1.0 \mathrm{~g} / \mathrm{cm}^{3}$, which is appropriate for tissue-equivalent gels [7].

\section{RESULtS}

Fig. 2, 3, and 4 show the wave images at $50 \mathrm{~Hz}, 100 \mathrm{~Hz}$ and $200 \mathrm{~Hz}$ excitations respectively. Longitudinal motion of the acupuncture needle induced the shear waves in the gel phantom. As the excitation frequency increased, the wavelength of the shear waves decreased. Few image artifacts were observed near the needle.

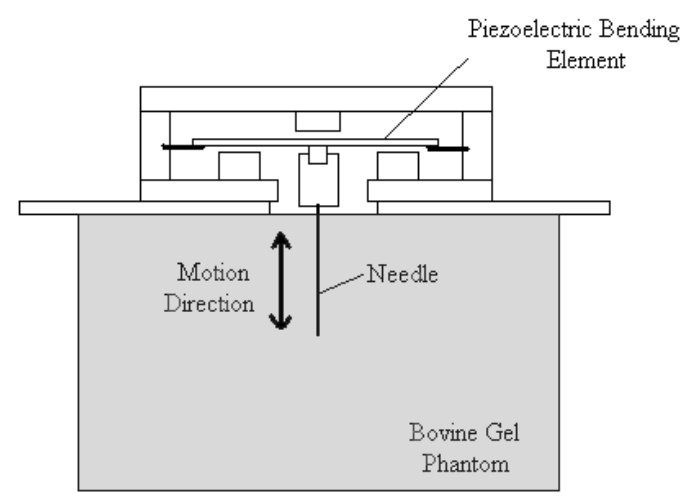

Fig. 1. The configuration of the piezoelectric driver 
The shear stiffness estimated from the wave images and the standard deviation of the shear stiffness in the gel phantom at $50 \mathrm{~Hz}, 100 \mathrm{~Hz}$ and $200 \mathrm{~Hz}$ are summarized in Table I.

\section{Discussion}

It has been shown that shear waves can be induced in vitro by rods, point sources or rectangular planes [1-6]. The size of these wave sources is larger than $100 \mathrm{mmx} 100 \mathrm{~mm}$. In this study, shear waves were generated in a bovine gel phantom using a $0.4 \mathrm{~mm}$ diameter acupuncture needle. To the best of our knowledge, no previous reports are found that shear waves can be induced by a fine and sharp needle.

As the excitation frequency increases from $50 \mathrm{~Hz}$ to $200 \mathrm{~Hz}$, the wavelength in the gel phantom decreases. This phenomenon agrees with the wave propagation properties. The wavelength at $50 \mathrm{~Hz}$ excitation frequency cannot be measured from the wave image, as the wavelength was longer than the width of the gel phantom. If the gel phantom is replaced by selected muscle group in vivo, the waves will propagate along the muscle length. The wavelength at low frequencies may be more readily measured in vivo, as they will be comparatively longer. Shear waves induced by a moving acupuncture needle can directly apply to the region of interest deeply embedded inside the object. Using the acupuncture needle to generate the shear waves may be suitable for the study at arm and leg muscles since the propagation direction of the waves is parallel to the muscle fiber direction.

In bovine gels the viscosity is a function of the excitation frequency [8]. The shear stiffness values at different excitation frequencies were not the same due to the dispersive nature of bovine gel phantoms. Further study is planned to observe the wave motion inside an in vitro bovine muscle specimen.

Table I

Shear stiffness estimated and standard deviation

\begin{tabular}{ccc}
\hline $\begin{array}{c}\text { Excitation } \\
\text { Frequency }\end{array}$ & Shear stiffness & Standard deviation \\
\hline $50 \mathrm{~Hz}$ & $11.388 \mathrm{kPa}$ & 3.914 \\
$100 \mathrm{~Hz}$ & $7.505 \mathrm{kPa}$ & 1.573 \\
$200 \mathrm{~Hz}$ & $9.251 \mathrm{kPa}$ & 2.015 \\
\hline
\end{tabular}

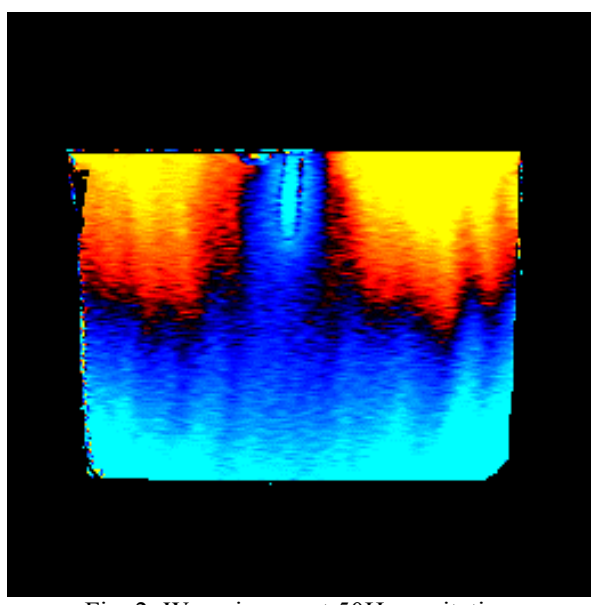

Fig. 2. Wave image at $50 \mathrm{~Hz}$ excitation

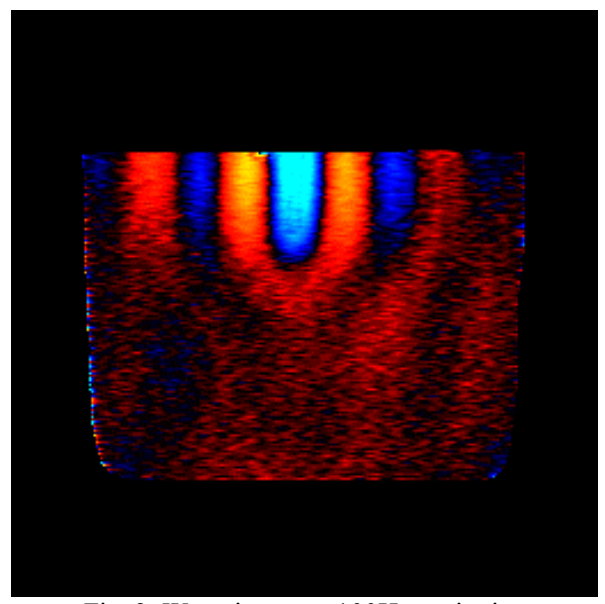

Fig. 3. Wave image at $100 \mathrm{~Hz}$ excitation

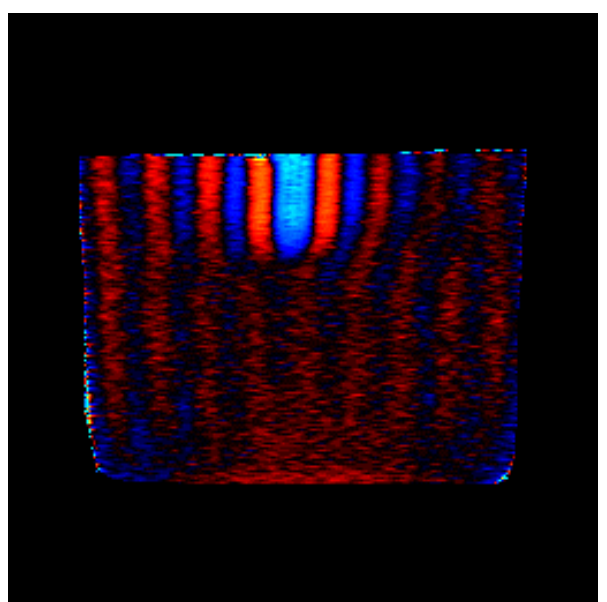

Fig. 4. Wave image at $200 \mathrm{~Hz}$ excitation 


\section{CONCLUSION}

The experimental results show that it is possible to generate propagating waves using a moving acupuncture needle and observe the phenomenon with MRE. In previous works, in vivo studies were limited to shear waves generated at the surface of the tissue [1-5]. A disadvantage of this approach is that shear waves can be heavily attenuated by tissue on the surface, and can have difficulties reaching deeper structures of interest. By inserting the needle into the muscle, the shear waves will be applied directly to the muscle of interest. It appears that the induced waves by a moving needle can be used to study the mechanism of acupuncture in vivo with MRE. The physical acupuncture treatment can be simulated by moving the needle up and down (in and out) at a specific acupoint. Further study will be conducted to verify the use of moving needle in an in vivo study.

\section{ACKNOWLEDGMENT}

This study was supported by the Hong Kong Jockey Club Charities Trust.

\section{REFERENCES}

[1] R. Muthupillai, D. J. Lomas, P. J. Rossman, J. F. Greenleaf, A. Manduca and R. L. Ehman, "Magnetic resonance elastography by direct visualization of propagating acoustic strain waves," Science, vol. 269, pp. 1854-1857, September 1995.

[2] R. Muthupillai, P. J. Rossman, D. J. Lomas, J. F. Greenleaf, S. J. Riederer and R. L. Ehman, "Magnetic resonance imaging of transverse acoustic strain waves," Magn. Reson. Med., vol. 36, pp. 266-274, August 1996.

[3] M. A. Dresner, G. H. Rose, P. J. Rossman, R. Muthupillai, A. Manduca and R. L. Ehman, "Magnetic resonance elastography of skeletal muscle," JMRI, vol. 13, pp. 269-276, February 2001.

[4] G. Heers, T. Jenkyn, M. A. Dresner, M. O. Klein, J. R. Basford, K. R. Kaufman, R. L. Ehman and K. N. An, "Measurement of muscle activity with magnetic resonance Elastography," Clin. Biomechaincs, vol. 18, pp. 537-542, July 2003.

[5] A. Manduca, T. E. Oliphant, M. A. Dresner, J. L. Mahowald, S. A. Kruse, E. Amromin, J. P. Felmlee, J. F. Greenleaf and R. L. Ehman, "Magnetic resonance elastography: non-invasive mapping of tissue elasticity," Medical Image Analysis, vol. 5, 237-254, December 2001.

[6] P. Rossman, K. Glaser, J. Felmlee and R. Ehman, "Piezoelectric bending elements for use as motion actuators in MR Elastography," Proc. Intl. Soc. Mag. Reson. Med., vol. 11, pp. $1075,2003$.

[7] M. M. Burlew, E. L. Madsen, J. A. Zagzebski, R. A. Banjavic and S. W. Sum, "A new ultrasound tissue-equivalent material," Radiology, vol. 34, pp. 517-520, 1980.

[8] S. A. Kruse, J. A. Smith, A. J. Lawrence, M. A. Dresner, A. Manduca, J. F. Greenleaf and R. L. Ehman, "Tissue characterization using magnetic resonance elastography: preliminary results," Phys Med Biol., vol. 45, pp. 1579-90, June 2000. 